\title{
Modeling and Application of Pedestrian Safety Conflict Index at Signalized Intersections
}

\author{
Wei Cheng, ${ }^{1}$ Ning Zhang, ${ }^{2}$ Wei $\mathrm{Li}^{3}{ }^{3}$ and Jianfeng $\mathrm{Xi}^{3}$ \\ ${ }^{1}$ School of Transportation Engineering, Kunming University of Science and Technology, Kunming, Yunnan 650500, China \\ ${ }^{2}$ College of Science and Engineering, University of Minnesota, Minneapolis, MN 55455, USA \\ ${ }^{3}$ School of Transportation, Jilin University, Changchun, Jilin 130022, China
}

Correspondence should be addressed to Wei Cheng; chengwei_ding@163.com

Received 26 January 2014; Revised 17 February 2014; Accepted 23 February 2014; Published 31 March 2014

Academic Editor: Wuhong Wang

Copyright (C) 2014 Wei Cheng et al. This is an open access article distributed under the Creative Commons Attribution License, which permits unrestricted use, distribution, and reproduction in any medium, provided the original work is properly cited.

\begin{abstract}
Traffic conflict between turning vehicles and pedestrians is the leading cause of pedestrian fatalities at signalized intersections. In order to provide a solution for evaluating intersection safety for vulnerable road users, this paper first determines the most important factors in analyzing pedestrian-vehicle conflict and puts forward a pedestrian safety conflict index (SCI) model to establish a quantitative standard for safety evaluation of two- or multiphase intersections. A safety level system is then designed based on SCI to help categorize and describe the safety condition of intersections applicable to the model. Finally, the SCI model is applied to the evaluation of two intersections in the city of Changchun, the result of which complies with expectation, indicating the model's potential in providing an improved approach for pedestrian-vehicle conflict evaluation study.
\end{abstract}

\section{Introduction}

Pedestrian traffic comprises a large proportion of the total mixed traffic flow at urban intersections in China $[1,2]$. Crash data consistently show that collisions with pedestrians occur far more often with turning vehicles than with straightthrough traffic [3,4]. Conflict between crossing pedestrians and left-turn vehicles is the leading cause of pedestrianrelated crashes [5]. Left-turn vehicles are more often involved in pedestrian accidents than right-turn vehicles, partly because drivers are not able to see pedestrians to the left as well $[6,7]$. Therefore, it bares great importance to study the mechanism of left-turn vehicle and pedestrian conflict and establish sound safety evaluation systems in order to better protect vulnerable road users and develop a safer and more efficient road traffic environment.

Two prominent methods for evaluating traffic safety at intersections are (1) direct evaluation based on traffic conflict and (2) indirect evaluation based on traffic accident [811]. When conducting traffic conflict research with regard to pedestrian-vehicle safety evaluation, some researchers choose to calculate one or more traffic conflict index numbers through processing field data and use the index numbers as the basis for intersection safety evaluation [12], whereas others would approach the same problem following mainly two paths, the first of which is by devising a conflict probability model $[11,13]$ and the second by establishing a conflict index (or hazard index) [14-16]. In either case, the majority of the researches are based on the premise of acquiring traffic conflict data beforehand. However, traffic data collection in China, at the moment, mainly depends on subjective observation and judgment from survey conductors. Human factor is one major reason for the lack of consistency when integrity of data is concerned.

This paper introduces the concept of pedestrian safety conflict index and establishes a safety level system for evaluating the safety of crossing pedestrians based on the study of the mechanism of left-turn vehicle and pedestrian conflict at signalized intersections, with the hope of providing an improvement to pedestrian safety evaluation at signalized intersections. 


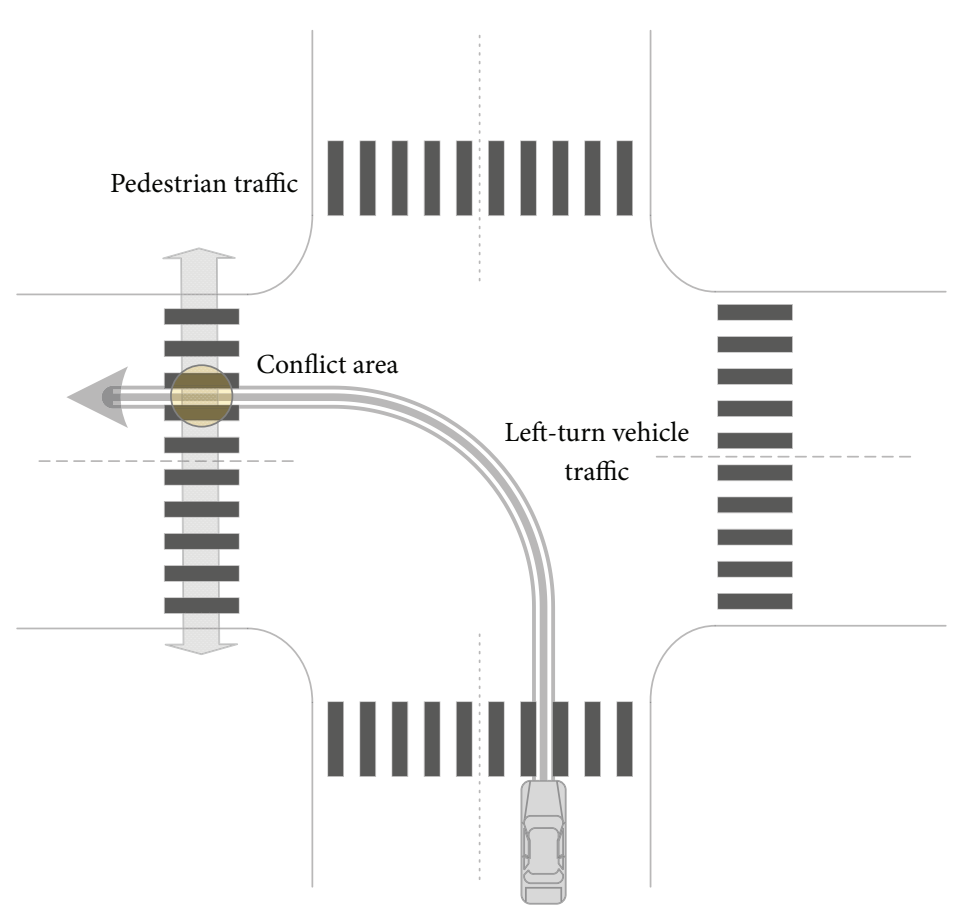

FIGURE 1: Illustration of left-turn vehicle and pedestrian conflict.

\section{Mechanism of Left-Turn Vehicle and Pedestrian Conflict}

At intersections that allow left turning, left-turning vehicles often start at the same moment as or shortly after (e.g., leading pedestrian interval) through traffic and crossing pedestrians, which leads to conflict between turning vehicles and pedestrians, as illustrated in Figure 1.

The conflict area can be defined as an area in which collisions may occur if pedestrians and vehicles maintain their respective original state of movement.

As a left-turn vehicle approaches the crosswalk, its path intersects with crossing pedestrians walking in both directions. If both parties keep their respective original state of movement, that is no deceleration, acceleration or bypassing maneuvers, they would collide within the conflict area, as shown in Figure 2. $D_{x(v)}$ is the distance between the nearest side of the vehicle and curb; $D_{y(v)}$ is the distance between the front of the vehicle and the nearest side of the conflict area; and $D_{x(p)}$ is the distance between the crossing pedestrian and curb.

\section{Safety Analysis of Left-Turn Vehicle and Pedestrian Conflict}

3.1. Data Collection and Methodology. The data collection process is as follows, including the time and location chosen, as well as the methodology invested.

First, the collection of data was conducted through filming at all entrances and exits at three four-way signalized intersections in the city of Changchun. Four-way intersections make up the vast majority of signalized intersections in
China. Investigating as many types of intersection as possible is doable but unnecessary at this stage of the study. The intersections chosen are located at (A) West Democracy St.West Zhonghua Rd., (B) West Chaoyang Rd.-Construction St., and (C) Wanbao St.-Liberation Rd. The filming was carried out on two consecutive weekdays, each with 6 hours of actual filming time at 7:00-9:00 a.m. in the morning, 11:00 a.m. $-13: 00$ p.m. at noon, and 16:00-18:00 p.m. in the afternoon.

During the collection, action that meets at least one of the following categories was defined as one conflict: (1) pedestrian decelerates or halts to dodge vehicle; (2) pedestrian accelerates to dodge vehicle; (3) pedestrian bypasses to dodge vehicle; (4) vehicle decelerates or halts to dodge pedestrian; (5) vehicle accelerates to dodge pedestrian; (6) vehicle bypasses to dodge pedestrian.

In determining the factors of conflict, this paper focuses on pedestrian volume, density, average travel speed, and the volume and ratio of left-turn vehicles. All data is collected in Table $1 . C_{v}$ is number of left-turn vehicle and pedestrian conflicts; $Q_{p}$ is pedestrian crossing volume per hour; $K_{p}$ is dynamic pedestrian density; $V_{p}$ is pedestrian travel speed; $Q_{v}$ is volume of left-turn vehicles; $Q_{s}$ is volume of through traffic; $V_{v}$ is average spot speed of left-turn vehicles.

3.2. Design of Pedestrian Safety Conflict Index. Among the many factors involved in analyzing left-turn vehicle and pedestrian conflict, some have determining importance, while others only play insignificant roles that can be ignored. This paper analyzes the data in Table 1 to examine the importance of each individual conflict factor and to determine the most important ones. 


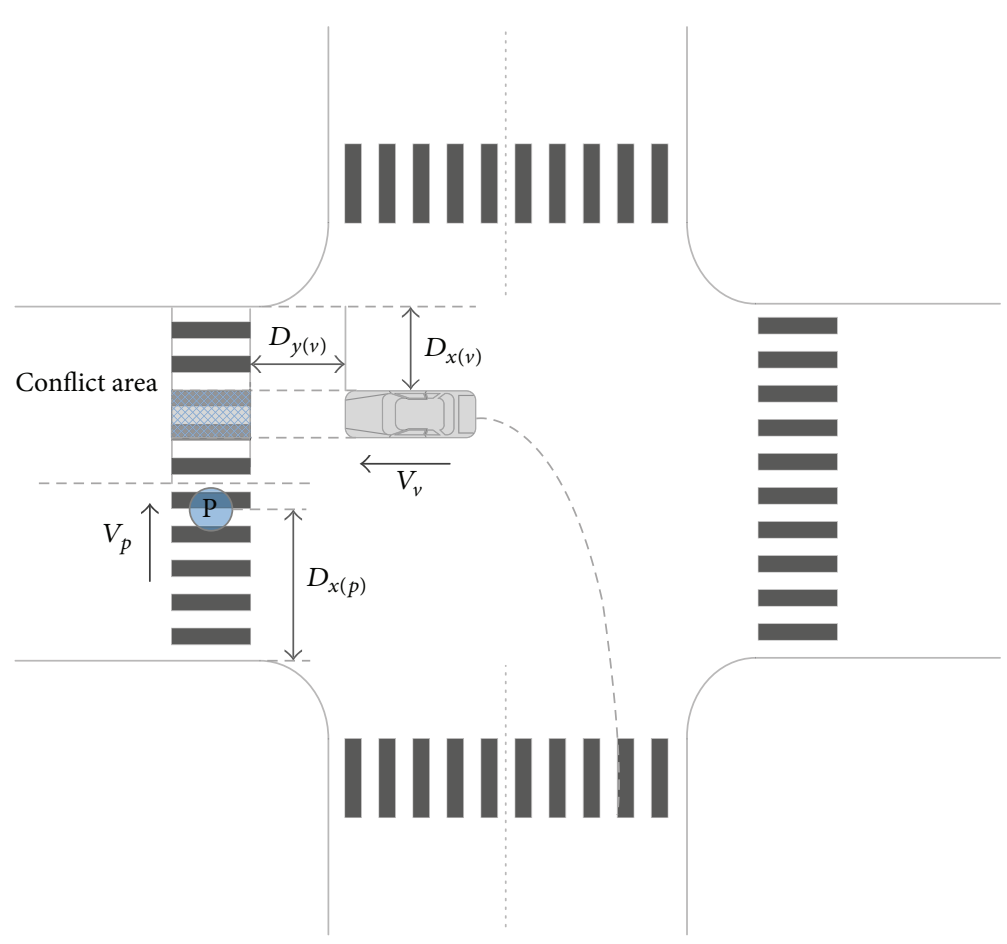

FIGURE 2: Illustration of conflict area.

TABLE 1: Table of collected data.

\begin{tabular}{|c|c|c|c|c|c|c|c|c|}
\hline Number & Intersection crossing & $C_{v}$ perhr & $Q_{p}(\mathrm{p} / \mathrm{h})$ & $K_{p}\left(\mathrm{p} / \mathrm{m}^{2}\right)$ & $V_{p}(\mathrm{~m} / \mathrm{s})$ & $Q_{v}(\mathrm{Veh} / \mathrm{h})$ & $Q_{s}(\mathrm{Veh} / \mathrm{h})$ & $V_{v}(\mathrm{~km} / \mathrm{h})$ \\
\hline 1 & A, morning peak & 15 & 129 & 0.1185 & 1.21 & 84 & 398 & 20.96 \\
\hline 2 & A, noon peak & 41 & 136 & 0.1326 & 1.14 & 71 & 328 & 19.36 \\
\hline 3 & A, afternoon peak & 38 & 158 & 0.1554 & 1.13 & 66 & 382 & 22.82 \\
\hline 4 & $\mathrm{~B}$, morning peak & 23 & 121 & 0.1430 & 1.41 & 77 & - & 23.80 \\
\hline 5 & $\mathrm{~B}$, noon peak & 19 & 108 & 0.1295 & 1.39 & 98 & - & 24.76 \\
\hline 6 & B, afternoon peak & 98 & 153 & 0.2107 & 1.21 & 197 & - & 25.64 \\
\hline 7 & $\mathrm{C}$, morning peak & 30 & 141 & 0.1618 & 1.13 & 67 & 314 & 21.70 \\
\hline 8 & C, noon peak & 26 & 83 & 0.0996 & 1.08 & 50 & 325 & 21.80 \\
\hline 9 & $\mathrm{C}$, afternoon peak & 47 & 169 & 0.1872 & 1.17 & 72 & 327 & 22.12 \\
\hline
\end{tabular}

Many of the major factors can be extracted from Table 1 directly, such as $C_{v}, Q_{p}, V_{p}$, and $Q_{v}$. Here introduced the pedestrian coefficient of spatial distribution [11], represented as $D$ :

$$
D=\frac{1}{K_{p}},
$$

and disadvantaged groups percentage, represented as DG. $N_{\text {DG }}$ is the number of elderly and underage crossing pedestrians per unit time, and $N_{t}$ is the number of total crossing pedestrians per unit time; therefore

$$
\mathrm{DG}=\frac{N_{\mathrm{DG}}}{N_{t}} .
$$

In order to simplify the calculation and analysis process, units of pedestrian travel speed and vehicle spot speed are both converted to $\mathrm{km} / \mathrm{h}$, using the following equations:

$$
V_{p}^{\prime}=3.6 \times V_{p}, \quad V_{v}^{\prime}=3.6 \times V_{v} .
$$

A multiple linear regression calculation was conducted on $C_{v}, Q_{p}, V_{p}^{\prime}, Q_{v}, D$, and DG. And a conclusion was drawn through an analysis of each factor's coefficient, the result of which is that left-turn vehicle volume has the most influence on conflict, with a coefficient of 0.811 . The other two determining factors are the volume and density of crossing pedestrians. On the other hand, variations in speed factors, including pedestrian travel speed and left-turn vehicle speed, made little difference to the final result. If any factor does not meet the significance standard, another regression should be done with speed factors removed. In the test, $R^{2}$ returns a value of 0.893 , which implies that the result is desirably fitted. However, the degree of significance is 0.075 , larger than 0.5 , rendering the regression equation invalid [12]; therefore, there is no need to conduct further significance tests. 
Regression calculation with speed factors removed returns a value of 0.87 for $R^{2}$, which is larger than 0.85 , and the overall significance is 0.012 , smaller than 0.05 , rendering the regression valid. Regression equation is shown in the following equation:

$$
\begin{aligned}
C_{v}= & -257.54+0.327 Q_{p}+811.596 K_{p}+15.656 D \\
& +0.324 Q_{v}+0.575 D G .
\end{aligned}
$$

Equation (4) is the result of regression; to make sure it is greater than 0 , reflecting the number of conflicts in the real word, the values of $Q_{p}$ and $Q_{v}$ should always be actual volumes of pedestrian and turning vehicles.

Pedestrian safety conflict index (SCI) was designed based on conflict analysis of left-turn vehicles and pedestrians at signalized intersections, the range of which is 0 to 1 . The purpose of SCI is to provide a system of standards for evaluating signalized intersection safety; the higher the index number is, the safer the pedestrians are, when crossing streets.

When establishing the SCI model, (4) can be simplified. From the previous regression, the relation coefficient of $Q_{p}$ and $D$ is -0.817 , with a degree of $80 \%$; therefore, after another regression, a new equation with $Q_{p}$ and $D$ is as follows:

$$
D=-7.72 \ln Q_{p}+45.22 .
$$

Also because

$$
K_{p}=\frac{1}{D}=\frac{1}{-7.72 \ln Q_{p}+45.22}
$$

equation (4) can be transformed to

$$
\begin{aligned}
C_{v}= & \frac{933.1\left(\ln Q_{p}\right)^{2}-8942.8 \ln Q_{p}+20368.2}{-7.72 \ln Q_{p}+45.22} \\
& +0.327 Q_{p}+0.324 Q_{v}+0.575 \mathrm{DG} .
\end{aligned}
$$

From (7), we can draw the following conclusion that conflict between left-turn vehicles and pedestrians at two-phase intersections is determined by 3 main factors: pedestrian volume, left-turn vehicle volume, and disadvantaged group factor. Their coefficients can be represented by constants $a, b$, $c$, and $d$; hence there is

$$
C_{v}=a \cdot Q_{p}+b \cdot Q_{v}+c \cdot D G+d .
$$

SCI, being established with the purpose of evaluating safety conditions of signalized intersections-the higher the value, the less the incidents of conflict and the safer the intersection - is calculated as follows:

$$
\mathrm{SCI}=A^{C_{v}}=A^{a \cdot Q_{p}+b \cdot Q_{v}+c \cdot D G+d}, \quad A \in(0.1) .
$$

Coefficient $A$ is determined by SCI and the number of conflicts. All the surveyed crossings are on service level $A$; therefore the defined SCI index number should be larger than $80 \%$. The left-turn vehicle and pedestrian conflict at the three data-gathering periods after regression calculation are listed in Table 2.

In equation (9), $A$ equals 0.9971 when $\mathrm{SCI}$ is given 0.8 and the maximum number of conflicts is 77 .

The relation between SCI and the number of conflicts is (10) and shown in Figure 3. Consider the following:

$$
\mathrm{SCI}=0.9971^{C_{\nu}} .
$$

Bringing (7) into (10), SCI can be expressed as follows:

$$
\mathrm{SCI}=0.9971^{\left(\left(933.1\left(\ln Q_{p}\right)^{2}-8942.8 \ln Q_{p}+20368.2\right) /\left(-7.72 \ln Q_{p}+45.22\right)\right)+0.327 Q_{p}+0.324 Q_{\nu}+0.575 D G} .
$$

From the SCI model, it can be concluded that the volume of pedestrians and left-turn vehicles and the ratio of disadvantaged group to the crossing population play the most determinative roles in the study of pedestrian-vehicle conflict.

However, the model does not apply to all types of signalized intersections. When the number of pedestrians is fixed, as the number of left-turn vehicles increases, SCI decreases, but when the number of turning vehicle is fixed, even if pedestrian number increases, SCI decreases at first instead of increasing. And only when the number of pedestrians reaches $115 \mathrm{p} / \mathrm{h}$, SCI complies with the result of the survey. So $Q_{p} \geq$ $115 / \mathrm{h}$ is always needed for the model to work.

In conclusion, the four requirements of the SCI model are as follows. (1) Arrival of pedestrians and vehicles should be random. This is due to the fact that if the traffic flow is continuous the behavioral and psychological activities of pedestrians will be affected, causing inconsistency between the model's perdition and survey observation. (2) Pedestrian volume should be at least $115 \mathrm{p} / \mathrm{h}$. (3) Right-turning vehicles should have limited to no interference with the crossing pedestrians. The SCI model was established with the premise of ignoring right-turn interference, so the model does not apply to heavily interfered intersections. (4) The model only applies to two- or multiphase signalized intersections where the majority of crossing pedestrians abide by traffic rules and where there are no severe traffic law violations.

3.3. Intersection Safety Levels Based on SCI. When conditions of SCI model are met and the model applied to two- or multiphase intersections, the safety condition of an intersection can be evaluated with SCI. 
TABLE 2: Regression table of left-turn vehicle and pedestrian conflict.

\begin{tabular}{lccccccccc}
\hline Number & 1 & 2 & 3 & 4 & 5 & 6 & 7 & 8 & 9 \\
\hline$C_{v}$ (rounded) & 25 & 31 & 41 & 22 & 35 & 77 & 26 & 23 & 49 \\
\hline
\end{tabular}

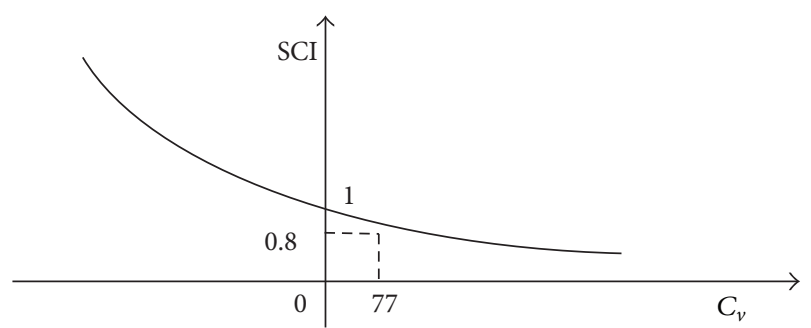

FIgURE 3: Graph of relationship between SCI and $P-V$ conflict.

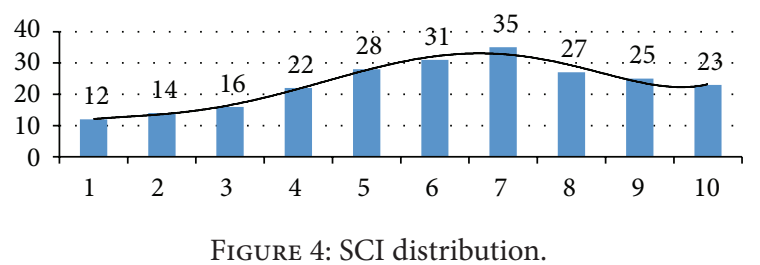

The SCI distribution is calculated by bringing $Q_{p}, Q_{v}$, and DG values into the SCI equation, and the distribution is shown in Figure 4.

As the figure illustrates, SCI follows the normal distribution, which agrees with expectation and survey data. Based on the distribution, SCI can be divided into 4 levels, described in Table 3.

The 4-leveled system can provide a quantitative measurement for the evaluation of crossing pedestrians at intersections.

\section{Application}

The SCI model was applied to the safety evaluation of intersections on West Chaoyang Rd.-Construction St. and Free Rd.-Comrade St. Time of survey was during morning and afternoon peak hours, with 15 minutes of filming intervals. The whole process lasted for 2 hours. Data is shown in Table 4.

And the SCIs of the two intersections are

$$
\mathrm{SCI}_{1}=0.8787 ; \quad \mathrm{SCI}_{2}=0.7014
$$

According to Table 3, the safety level at the West Chaoyang rd.-Construction St. intersection is A, and B at the Free Rd.-Comrade St. Therefore, a theoretical conclusion is that the former intersection is safer for pedestrians to cross than the latter. As a matter of fact, the Free Rd.-Comrade St. intersection is situated in a population-dense area, with great volume of vehicles and pedestrians. And there are no dedicated signals for pedestrians, causing frequent violations of traffic rules, increasing the possibility of conflict and danger.
TABLE 3: Safety level of pedestrians.

\begin{tabular}{|c|c|c|}
\hline SCI & Safety level & Description \\
\hline$>0.8$ & A & Very few conflicts, very safe \\
\hline $0.5-0.8$ & B & $\begin{array}{l}\text { Relatively severe conflicts, potential } \\
\text { danger }\end{array}$ \\
\hline $0.3-0.5$ & $\mathrm{C}$ & $\begin{array}{l}\text { Severe conflicts, intersection redesign } \\
\text { required }\end{array}$ \\
\hline$<0.3$ & $\mathrm{D}$ & $\begin{array}{l}\text { Severe conflicts, great danger, redesign } \\
\text { required }\end{array}$ \\
\hline
\end{tabular}

TABLE 4: Factor comparison.

\begin{tabular}{lccc}
\hline Intersection & $Q_{p}(\mathrm{p} / \mathrm{h})$ & $Q_{v}(\mathrm{Veh} / \mathrm{h})$ & $\mathrm{DG}$ \\
\hline $\begin{array}{l}\text { (1) West Chaoyang } \\
\text { Rd.-Construction St. }\end{array}$ & 169 & 72 & 7.10 \\
(2) Free Rd.-Comrade St. & 220 & 105 & 14.10 \\
\hline
\end{tabular}

\section{Conclusions}

Conflict between left-turn vehicles and pedestrians at signalized intersections has increasingly become a safety threat to roadway traffic operation, especially in fast-developing countries like China. This paper, based on the study of pedestrian-vehicle conflict mechanism at signalized intersections and sufficient survey data, introduced the SCI model and safety level system, established a systematic and quantitative method of intersection safety evaluation from the perspective of traffic conflict.

Although there is still much space for improvement on the model, which requires pedestrian volume to be above 115 persons per hour and the intersection having limited to no interference from right-turning vehicles, the SCI model is applicable to the majority of city intersections which are mostly two- or multiphase, and the safety level system provides a quantitative way of categorizing the safety level of an intersection, as well as an intuitive way of describing their safety conditions. Together, the SCI model and the safety level system build a holistic framework for intersection safety evaluation and its study.

\section{Conflict of Interests}

The authors declare that there is no conflict of interests regarding the publication of this paper.

\section{References}

[1] Y.-L. Pei and S.-M. Feng, "Study on hazard degree of pedestrians' crossing based on traffic conflict," Journal of Harbin Institute of Technology, vol. 39, no. 2, pp. 285-287, 2007.

[2] J. Wang and S. Fang, "Pedestrian-vehicle conflict observation and characteristics of road section," Journal of Tongji University, vol. 36, no. 4, pp. 503-507, 2008.

[3] D. Lord, "Analysis of pedestrian conflicts with left-turning traffic," Transportation Research Record, no. 1538, pp. 61-67, 1996. 
[4] L. Ma and Z. F. Wang, "Intersection crossing safety analysis based on traffic conflict," Traffic Standardization, vol. 47, no. 11, pp. 94-97, 2011.

[5] J. Wang and S. Fang, "Pedestrian-vehicle conflict model and its application to pedestrian crossing street risk control," Journal of Tongji University, vol. 37, no. 9, pp. 1191-1195, 2009.

[6] K. L. Clark, J. E. Hummer, and N. Dutt, "Field evaluation of fluorescent strong yellow-green pedestrian warning signs," Transportation Research Record, no. 1538, pp. 39-46, 1996.

[7] N. Dutt, J. E. Hummer, K. L. Clark, and S. Blakely, "Controlledenvironment evaluation of fluorescent strong yellow-green pedestrian-crossing sign prototypes," Transportation Research Record, no. 1553, pp. 86-94, 1996.

[8] S. Ahuja, A. Chandra, T. Van Vuren, and M. G. H. Bell, "Pedestrian behavior and their perceptions around signalized traffic intersections," in Proceedings of the 11th World Conference on Transport Research (WCTR '07), Berkeley, Calif, USA, 2007.

[9] T. Sayed and S. Zein, "Traffic conflict models and standards for signalized and unsignalized intersections," in Proceedings of the Annual Conference of the Canadian Society for Civil Engineering, pp. 303-313, Halifax, Canada, June 1998.

[10] N. Harré and W. Wrapson, "The evaluation of a central-city pedestrian safety campaign," Transportation Research Part F: Traffic Psychology and Behaviour, vol. 7, no. 3, pp. 167-179, 2004.

[11] S. Zhou, K. P. Li, and J. Sun, "Simulation analysis of intersection conflict," China Safety Science Journal, vol. 19, no. 5, pp. 32-37, 2009.

[12] J.-J. Shen and W. Wang, "Vehicle-pedestrian conflict Probability Model Study," Journal of Transportation Systems Engineering and Information Technology, vol. 11, no. 1, pp. 152-156, 2011.

[13] J. Cinnamon, N. Schuurman, and S. M. Hameed, "Pedestrian injury and human behaviour: observing road-rule violations at high-incident intersections," PLoS ONE, vol. 6, no. 6, article e21063, 2011.

[14] Q. Li, Z. Wang, J. Yang, and J. Wang, "Pedestrian delay estimation at signalized intersections in developing cities," Transportation Research Part A: Policy and Practice, vol. 39, no. 1, pp. 61-73, 2005.

[15] J. Oxley, B. Fildes, E. Ihsen, J. Charlton, and R. Day, "Differences in traffic judgements between young and old adult pedestrians 1," Accident Analysis and Prevention, vol. 29, no. 6, pp. 839-847, 1997.

[16] C. Hydén, The development of a method for traffic safety evaluation: the Swedish Traffic Conflict Technique [doctoral thesis], Lund University, Department of Traffic Planning and Engineering, 1987. 


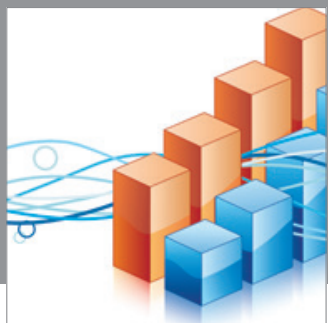

Advances in

Operations Research

mansans

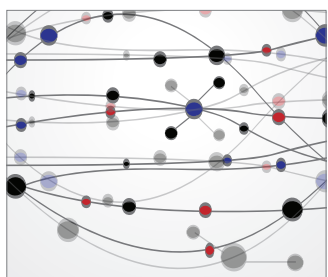

The Scientific World Journal
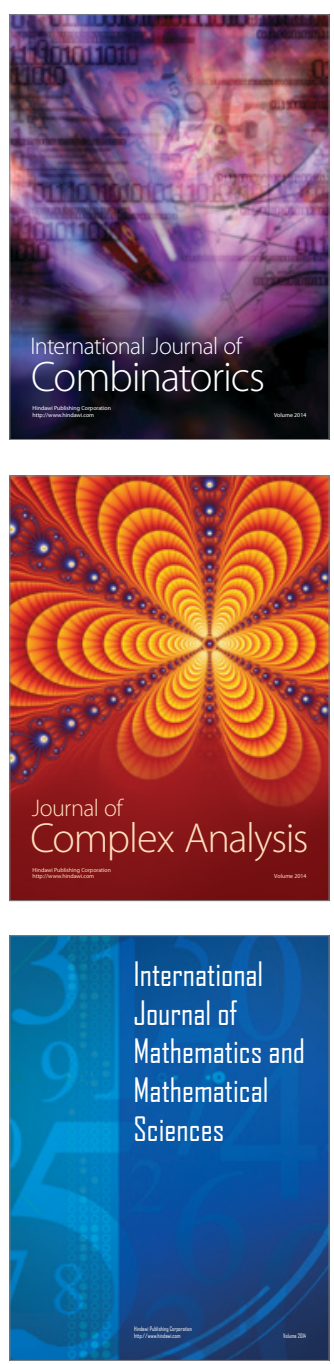
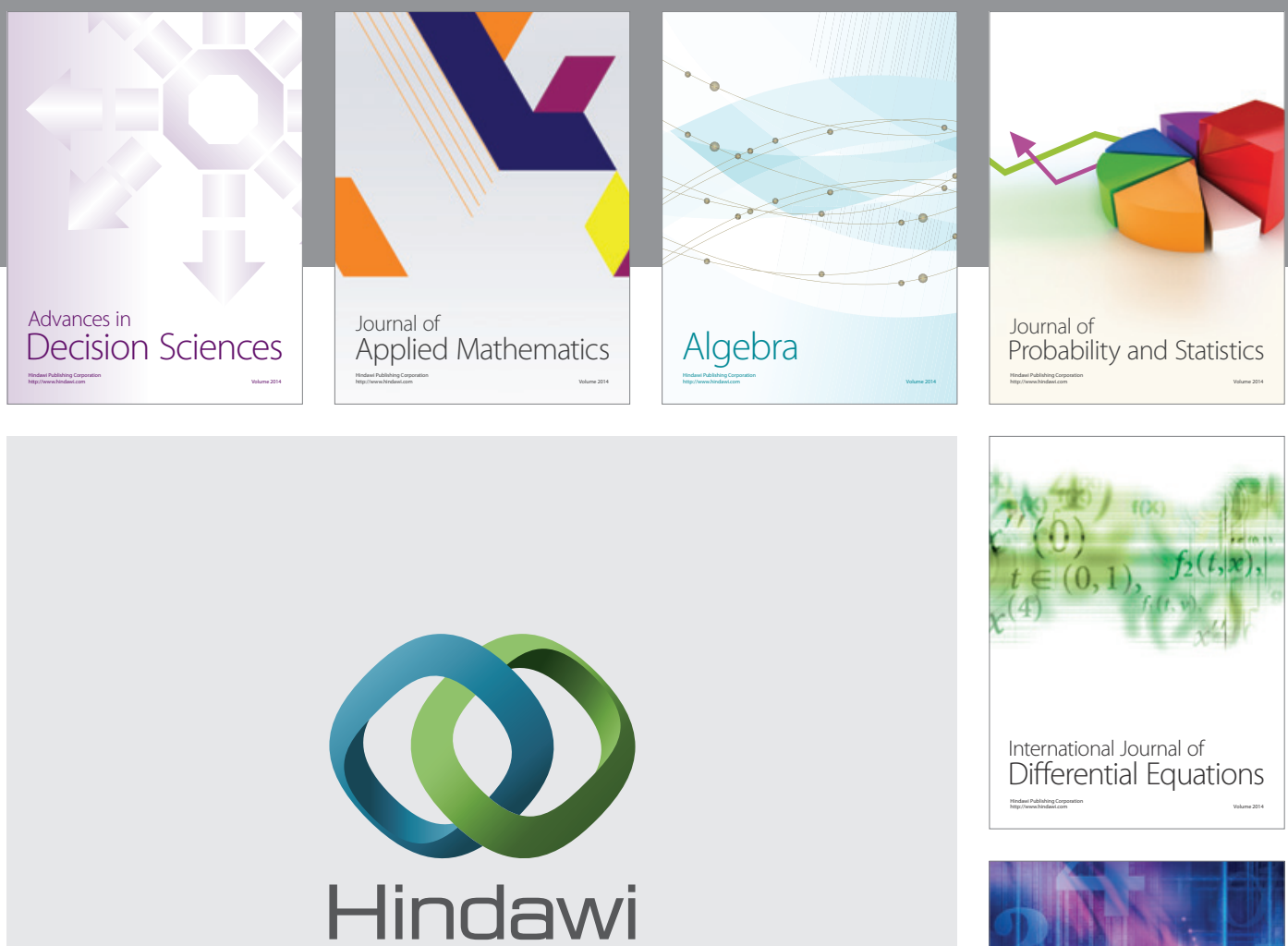

Submit your manuscripts at http://www.hindawi.com
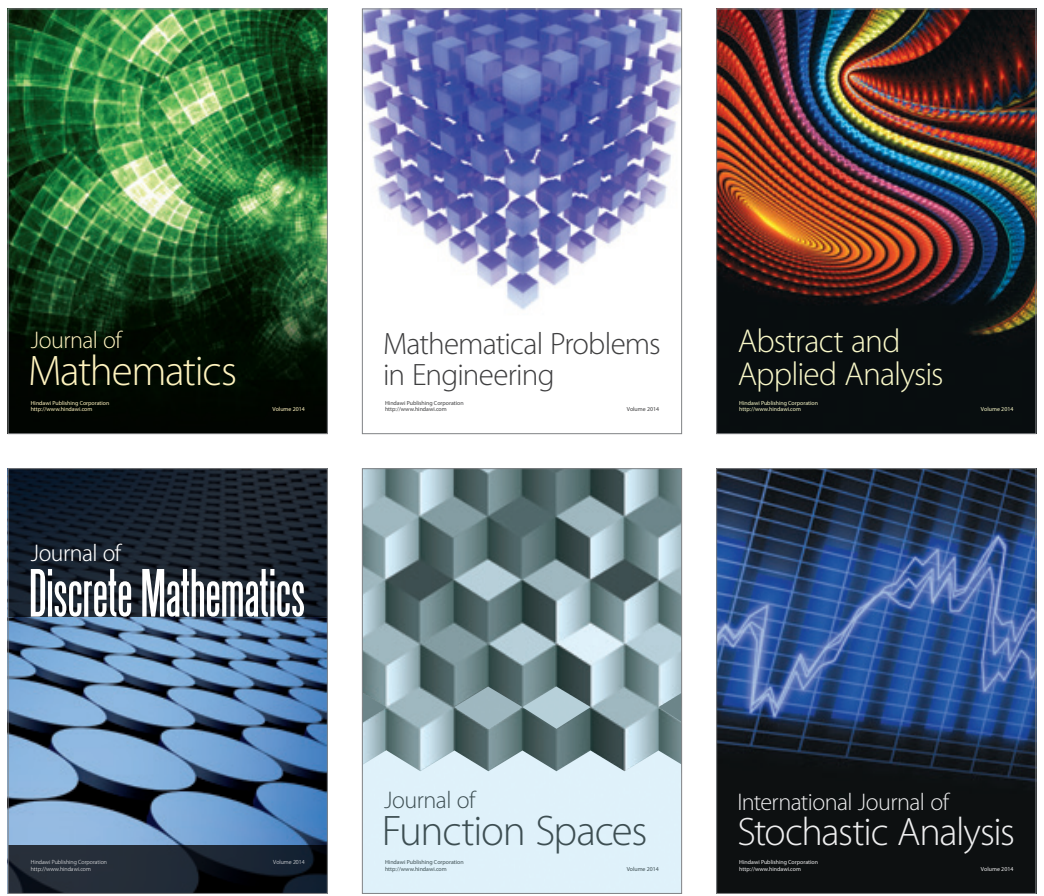

Journal of

Function Spaces

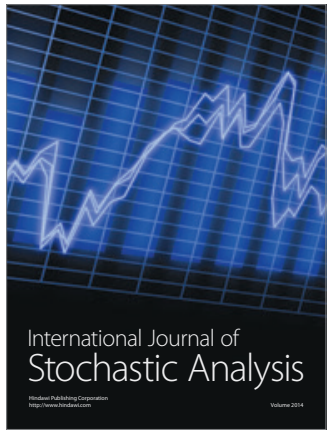

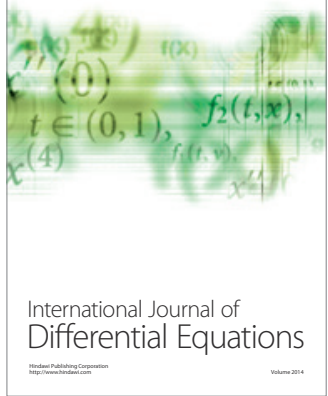
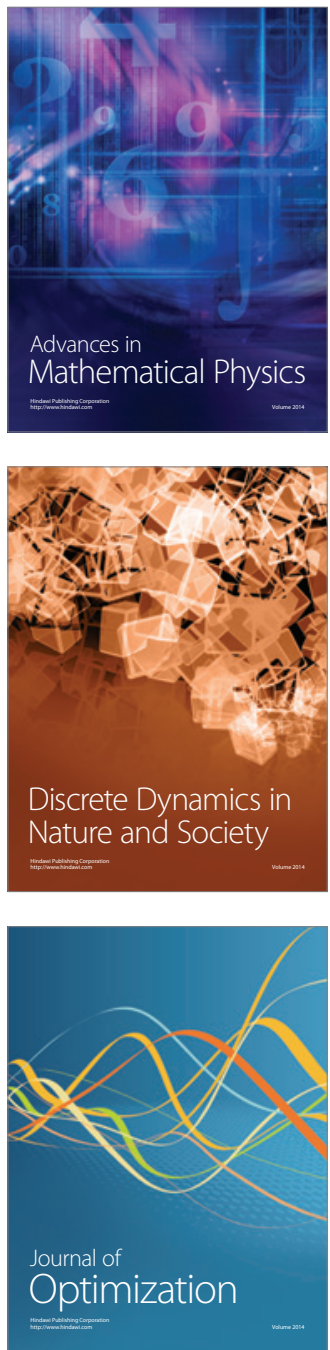